\title{
Development of a Coupled Subplane Capability in MPACT
}

\author{
Shane Stimpson, Aaron Graham, Benjamin Collins \\ Oak Ridge National Laboratory, P.O. Box 5800, Oak Ridge, TN 37831-6170
}

\begin{abstract}
Recent efforts in the development of the deterministic transport code MPACT have been devoted to preparing the $2 \mathrm{D} / 1 \mathrm{D}$ subplane method to be a production-level capability, as well as leveraging a multilevel coarse mesh finite difference (CMFD) approach to substantially reduce the runtime of target problems. For example, as compared to the previous default 2D/1D solver in MPACT on a standard quarter core model, the new solver reduces in core-hour requirements by $\sim 5-6 \times$.

Previous work focused solely on cases without multiphysics feedback, which is obviously important for analyzing the more realistic problems of operating reactors. The work presented in this article focuses on efforts to incorporate thermal hydraulics (TH) coupling through CTF by leveraging what are termed as subgrid solvers, which effectively treat material heterogeneities within subplane regions. Previous efforts have targeted using subgrid solvers for control rods and spacer grids; in this work, they are applied to account for the material property heterogeneities with regards to temperature/density distributions. This will allow the fidelity of coupling to be maintained while still reaping the performance benefits.
\end{abstract}

These new developments are demonstrated on two problems: (1) a single assembly case with feedback, known as Progression Problem 6a, and (2) a $3 \times 3$ cluster of assemblies with feedback based on Progression Problem $4 a$. The results demonstrate notable performance improvement potential for cases with TH feedback, but this approach is more dependent on the iteration process.

KEYWORDS: MPACT, MOC, 2D/1D, subplane, CTF

\section{INTRODUCTION}

In the past several years, work in MPACT [1], the primary deterministic transport solver in the Consortium for Advanced Simulation of Light Water Reactors (CASL) Virtual Environment for Reactor Analysis (VERA) [2], has focused on significantly improving the overall performance of the calculations, and many components within the code have been improved. More recently, efforts have been devoted to preparing the 2D/1D subplane method to be a production-level capability, as well as leveraging a multilevel coarse mesh finite difference (CMFD) approach to reduce the runtime of target problems $[3,4]$. For example, compared to the previous default 2D/1D solver in MPACT on a quarter core model, core-hour requirements were reduced by $\sim 5-6 \times$. However, subplane development has focused solely on cases without multiphysics feedback, which is obviously important for analyzing the more realistic problems of operating reactors.

The work in this article focuses on the development necessary to incorporate thermal hydraulics (TH) coupling through the CTF subchannel code [5]. MPACT/CTF coupling has been critical to the Consortium for Advanced Simulation of Light Water Reactors (CASL) mission and could significantly benefit from the runtime improvements possible using the subplane method. Previous work has dealt with the addition of subgrid solvers [6,7], which effectively treat material heterogeneities within subplane regions. While these previous efforts have targeted using subgrid solvers for control rods and spacer grids, they will be applied here to account for material property heterogeneities in temperature/density distributions, allowing the accuracy and fidelity of coupling to be maintained while capitalizing on subplane performance benefits.

This manuscript has been authored by UT-Battelle, LLC, under contract DE-AC05-00OR22725 with the US Department of Energy (DOE). The US government retains and the publisher, by accepting the article for publication, acknowledges that the US government retains a nonexclusive, paid-up, irrevocable, worldwide license to publish or reproduce the published form of this manuscript, or allow others to do so, for US government purposes. DOE will provide public access to these results of federally sponsored research in accordance with the DOE Public Access Plan (http://energy.gov/downloads/doe-public-access-plan). 


\section{SUBPLANE METHODOLOGY}

In the 2D/1D approach, 3D problems are decomposed into an axial stack of radial planes [1], where 2D method of characteristics (MOC) is used to solve radially [8], and 1D nodal methods operating on a homogenized pin-cell basis are used to solve axially [9]. The 2D and 1D solvers are coupled through transverse leakage, and CMFD is used to accelerate the global solution. MPACT used one-to-one mapping between the nodal and MOC solvers axially by default. However, a target axial node size of $\sim 8 \mathrm{~cm}$ can yield a substantial number $(\sim 55-60)$ of MOC planes

Originating from research performed using DeCART [10], the subplane method was used in the 2D/1D method in MPACT and could offer substantial reductions in computational burden. In the subject approach, multiple axial nodes are allowed to exist within each MOC plane allowing the axial solvers to be refined independently [10]. In the results shown herein, the subplane/nodal axial mesh is targeted to be very consistent with the axial meshes currently employed in MPACT, but with fewer MOC planes, the number of which depends on which material boundaries are simulated more explicitly.

Graham implemented the initial MPACT subplane capability [6], focusing mainly on ways to treat the control rod decusping problem which manifests itself when a control rod is partially inserted into an MOC plane. Graham also successfully developed several subgrid solver variants to treat rodded and unrodded regions of the MOC plane with a partially inserted rod to inform the coarse mesh cross sections used in the CMFD and 1D nodal axial sweepers.

\section{SUBGRID BACKGROUND}

In the subgrid approach, cross sections used in the CMFD and axial nodal solves were modified to more accurately represent material changes that could be occurring within each MOC plane. For example, in a more traditional subplane approach, spacer grids would be simulated explicitly with a separate MOC plane (and a subplane in between the spacers). However, by using subgrid, the spacer grid can be treated at the top of the MOC plane, where the subgrid solver handles the axial heterogeneity of the subplane nodes accurately, homogenizing as an approach for the MOC plane cross sections [4]. The same basic concept can be applied with feedback, except in this case each subplane level should be treated as a different cross section set based on changes to fuel, gap, clad, moderator temperatures, and moderator density (Figure 1).

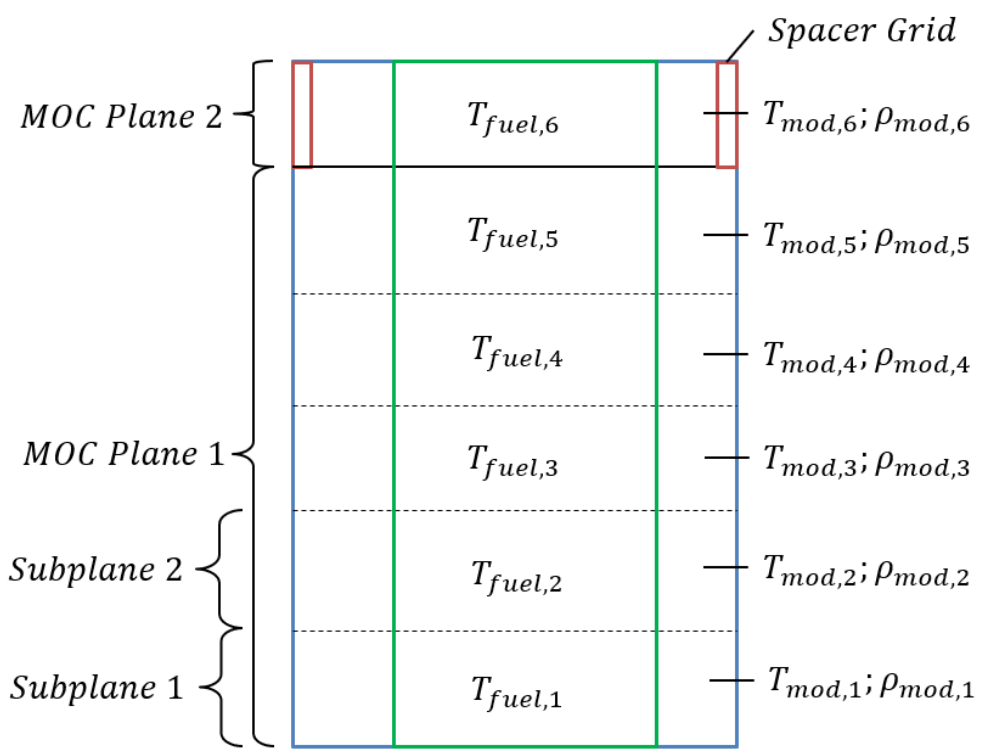

Figure 1. Subgrid Illustration Applied to TH Coupling. 
Here, we can see that the spacer grid is treated separately here to focus on the thermal hydraulic treatment, so subplane is only used between the spacer grids. This allows us to isolate the solution impact of treated feedback with subgrid from treating spacers with subgrid. In Figure 1, 5 subplanes are represented in MOC Plane 1, each with separate fuel and moderator temperatures/densities. For simplicity, clad and gap temperatures were neglected from the figure.

Two primary variants of subgrid solvers are relevant to this work (1) a subplane treatment in which axial cross section data within the MOC plane vary to correctly capture the material changes (rodded vs. unrodded or spacer grid vs. no grid), and (2) a collision probabilities treatment, which is an extension of the subplane treatment that employs a 1D collision probabilities method (CPM) solver for each disparate region of the subplane using the flux solution to better inform the coarse mesh-homogenized cross sections. More details on the subplane and collision probabilities treatments can be found in Graham's dissertation [2], but in this work, the subplane subgrid treatment is used exclusively.

\section{RESULTS}

\subsection{Overview of Cases Evaluated}

Results for two progression problems are shown here: (1) Problem 6a, Quarter Assembly, and (2) Problem 4a, now modified to include TH coupling [11]. To focus on the accuracy of the approximations imposed by the subplane method and the subgrid treatment of the spacer grids, the control rods were removed from all cases.

Six different solver configurations were evaluated for each of the following cases to assess the impact of relevant accuracy and performance. Effectively, the comparisons are subplane vs. default 2D/1D without subplane, and performance is evaluated with three different CMFD options (default, multilevel-in-space, and multilevel-in-energy):

1. Default: default mesh and solver options (explicit MOC planes for each axial level [58 total planes], a single level CMFD solver for all 51 groups, and pin-wise coarse mesh cells in the system)

2. Multilevel (ML) Space: same axial mesh as Default but using a multilevel-in-space CMFD solver

3. ML Energy: same axial mesh as Default but using a 3-level multilevel-in-energy CMFD solver; Level 1 has 51 groups, Level 2 has 10 groups, and Level 3 has 2 groups

4. Subplane: same CMFD option as Default, with explicit MOC planes for spacer grid, but with a single MOC plane between spacer grids, for a total of 23 MOC planes

5. Subplane+ML Space: same mesh as Subplane with ML Space CMFD solver

6. Subplane+ML Energy: same mesh as Subplane with ML Energy CMFD solver.

Additional details of the multilevel CMFD solvers is available in a companion paper to the conference [12], but effectively it is of interest to compare multiple CMFD options yield significant differences in runtime. For example, the load balancing during the CMFD solve can become suboptimal with various forms of subplane approximation and multilevel solvers (particularly in space, which includes an additional subplane spatial collapse) can help mitigate the performance burden of the load imbalance.

In these configurations, options 1-3 should yield identical solutions, as should options 4-6. However, the performance of each option varies. Essentially, options 1-3 all explicitly resolve all axial levels with an MOC plane and only vary in their CMFD options. Similarly 4-6 all use subplane treatment between spacer grids, and only vary in CMFD option. 


\subsection{Quarter Assembly with TH Coupling (Progression Problem 6a)}

Figure 2 shows the radial and axial layouts of the quarter assembly model, Progression Problem 6 [11]. It is a standard Westinghouse $17 \times 17$ assembly with 6 Zircaloy spacer grids in the active fuel and 2 Inconel grids, one at the bottom of the active fuel, and another in the upper reflector region. It is worth noting that it is entirely consistent with VERA Problem 3a, but it is simulated at $17.67 \mathrm{MWt}$ and $0.6823 \mathrm{Mlbs} / \mathrm{hr}$.
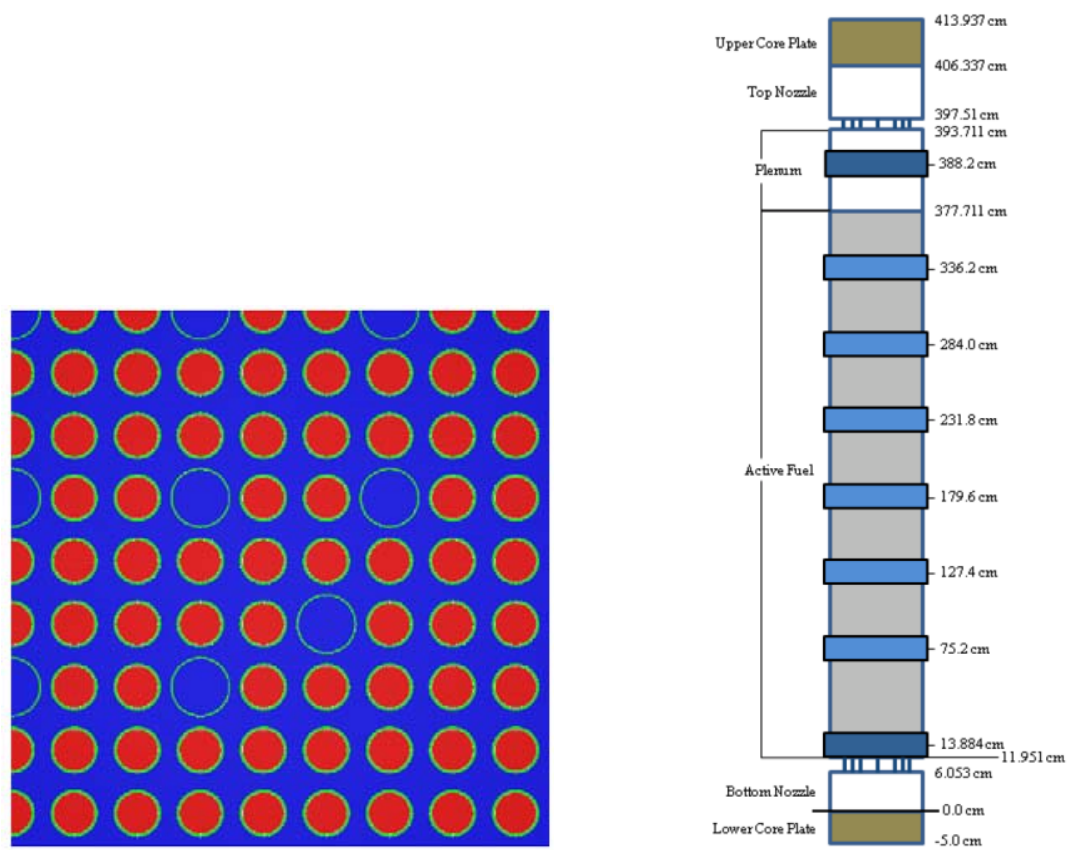

Figure 2. Problem 6a Specification [11].

Table I shows the solution differences for the 6 cases, with Default as the reference. In general, subplane does a very good job of sustaining the Default solution with a $5 \mathrm{pcm}$ eigenvalue difference and less than $0.15 \%$ power differences. While incorporating subgrid solvers introduces some error due to the approximations made to homogenized MOC cross sections and self-shielding, it is reasonable for the method. It can also be seen that the multilevel (ML) Space and ML Energy CMFD solvers yield similar results, with minor variation. This is related to the convergence criteria used by default in MPACT. The ML CMFD solvers change the path to solution, so the converged solution can deviate slightly from other cases in which the solution should be identical.

Table I. Problem 6a Accuracy Comparisons.

\begin{tabular}{|c|c|c|c|c|}
\hline Case & Eigenvalue & $\Delta \mathrm{k}(\mathrm{pcm})$ & $\begin{array}{c}\Delta \mathrm{P} \\
(\mathrm{RMS} \%)\end{array}$ & $\begin{array}{c}\Delta \mathrm{P} \\
(\mathrm{MAX} \%)\end{array}$ \\
\hline Default & 1.16550 & --- & --- & --- \\
\hline ML Space & 1.16550 & 0.01 & 0.020 & 0.031 \\
\hline ML Energy & 1.16550 & 0.25 & 0.028 & 0.058 \\
\hline Subplane & 1.16555 & 5.00 & 0.058 & 0.150 \\
\hline Subplane + ML Space & 1.16555 & 5.00 & 0.036 & 0.111 \\
\hline Subplane + ML Energy & 1.16555 & 5.00 & 0.035 & 0.116 \\
\hline
\end{tabular}


Figure 3 compares axial temperature distribution in the assembly (averaged radially) with Default and Subplane. The differences are less than a degree Celsius at any point. Figure 4 shows a similar comparison for the axial power distribution, with all differences $<0.15 \%$. There is a slight trend in the difference data, with underprediction of temperature near the bottom of the core and overprediction near the top.

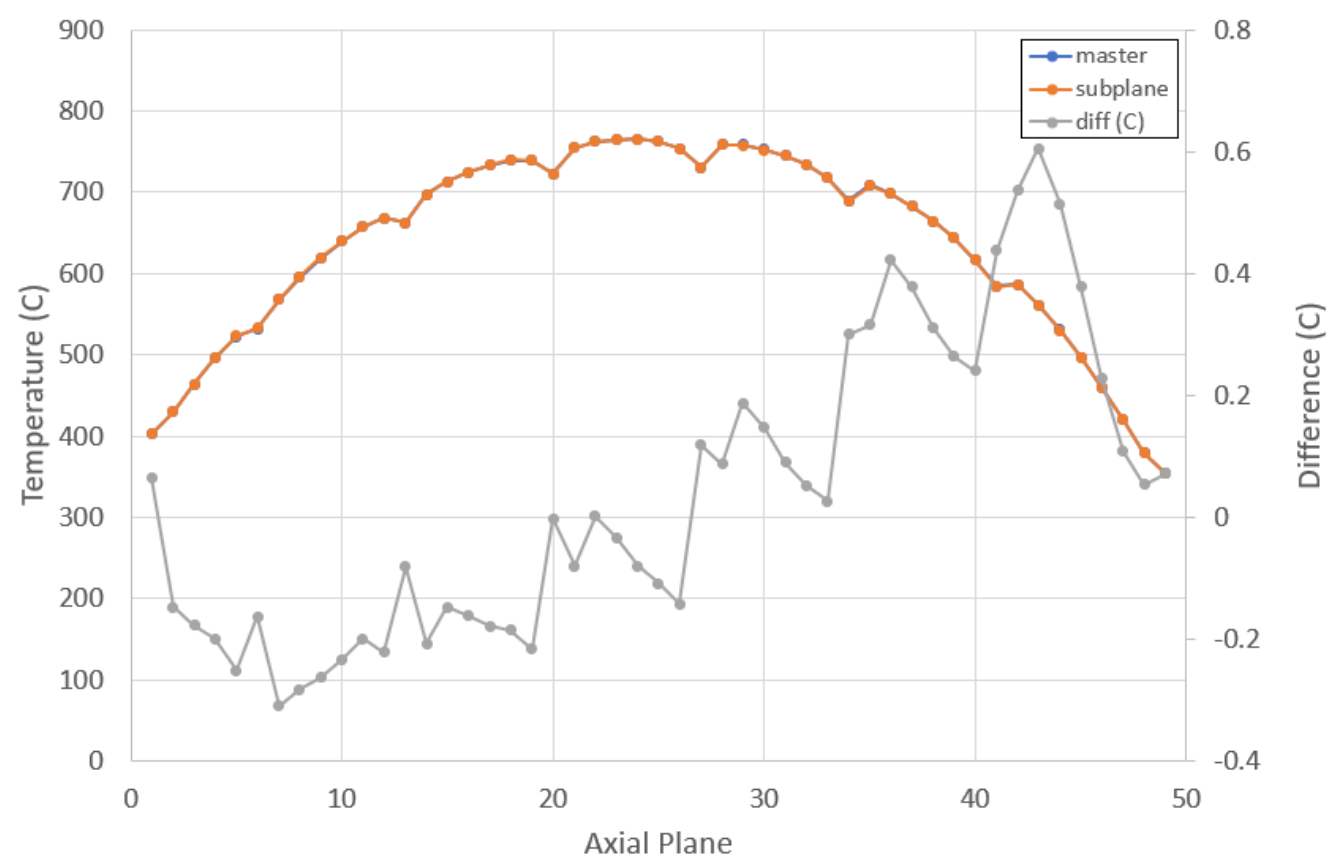

Figure 3. Problem 6a Subplane vs. Default Axial Temperature Comparison.

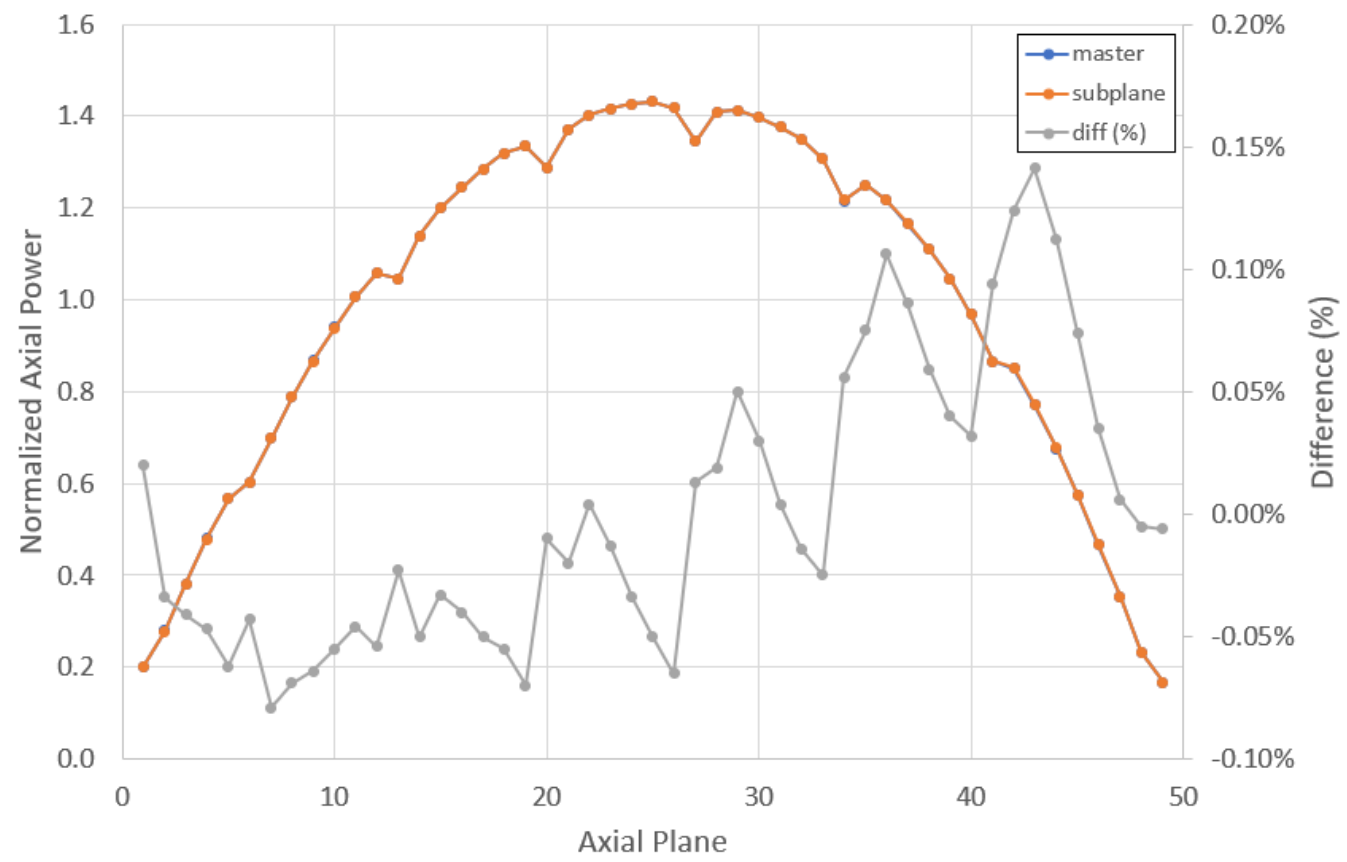

Figure 4. Problem 6a Subplane vs. Default Axial Power Comparison.

Table II shows pertinent performance data from 6a calculations. Full axial decomposition was used in all cases, resulting in 58 procs without subplane and 23 procs with subplane. Running with subplane incurs 
more outer iterations to converge the problem, which diminishes the runtime savings observed in cases without feedback, but overall, core-hours can still be reduced substantially from $\sim 16-10$ with subplane and the default CMFD and nearly 5 with subplane and ML Space CMFD.

Table II. Problem 6a Performance Comparisons.

\begin{tabular}{|c|c|c|c|c|c|}
\hline Case & Iterations & $\begin{array}{c}\text { Total } \\
\text { Memory } \\
(\mathrm{GB})\end{array}$ & $\begin{array}{c}\text { Walltime } \\
(\mathrm{min})\end{array}$ & Cores & $\begin{array}{c}\text { Time } \\
\text { (core hr) }\end{array}$ \\
\hline Default & 11 & 13.84 & 17.02 & 58 & 16.46 \\
\hline ML Space & 11 & 13.97 & 13.73 & 58 & 13.28 \\
\hline ML Energy & 12 & 14.14 & 11.79 & 58 & 11.40 \\
\hline Subplane & 20 & 6.95 & 25.84 & 23 & 9.90 \\
\hline Subplane + ML Space & 16 & 6.98 & 13.19 & 23 & 5.06 \\
\hline Subplane + ML Energy & 24 & 7.13 & 29.39 & 23 & 11.27 \\
\hline
\end{tabular}

It should also be noted that the performance gain from using subplane is also heavily mitigated because of the CTF runtime, which is a substantial portion of the total runtime. For example, in the ML space case, CTF took roughly 9 minutes of the 14 minute walltime and a similar amount of time in the subplane case.

\subsection{Colorset (Progression Problem 4a with Feedback)}

Problem 4a is a $3 \times 3$ assembly colorset featuring two enrichments with burnable poison rods (Figure 5). The axial layout can be found in Godfrey [11]. Similarly, all cases have 58 or 23 planes and processors/cores.

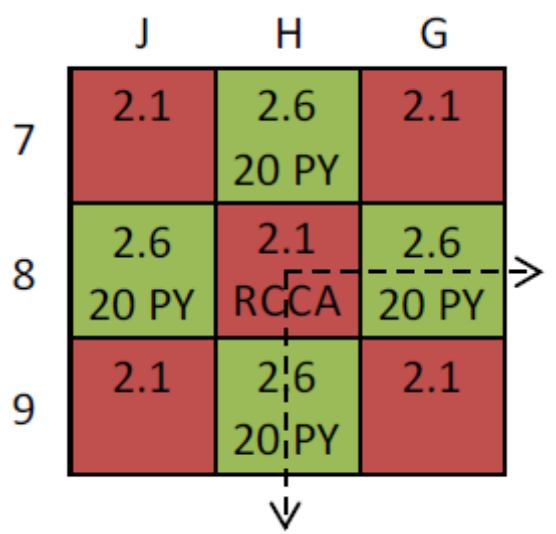

Figure 5. Problem 4a Colorset Radial Layout [11].

Table III shows the solution comparison for Problem 4a with feedback. In this case, slightly more notable solution differences are observed, and the maximum pin power errors are in the $0.5-0.6 \%$ range with subplane. In general, this is very good agreement, but some concern might be placed in how this error would propagate in depletion cases. 
Table III. Problem 4a Accuracy Comparisons.

\begin{tabular}{|c|c|c|c|c|}
\hline Case & Eigenvalue & $\Delta \mathrm{k}(\mathrm{pcm})$ & $\begin{array}{c}\Delta \mathrm{P} \\
(\mathrm{RMS} \%)\end{array}$ & $\begin{array}{c}\Delta \mathrm{P} \\
(\mathrm{MAX} \%)\end{array}$ \\
\hline Default & 0.98963 & --- & --- & --- \\
\hline ML Space & 0.98963 & 0.66 & 0.023 & 0.048 \\
\hline ML Energy & 0.98963 & 0.59 & 0.051 & 0.151 \\
\hline Subplane & 0.98969 & -5.35 & 0.169 & 0.552 \\
\hline Subplane + ML Space & 0.98969 & -5.40 & 0.196 & 0.596 \\
\hline Subplane + ML Energy & 0.98969 & -4.54 & 0.200 & 0.613 \\
\hline
\end{tabular}

Comparison data in Table IV show that while subplane provides a substantial memory reduction, the overall core-hour requirements are not substantially improved, as all core-hour requirements for the subplane cases increase. Detailed timing data suggest that much of the time for the subplane cases is still required in CMFD.

Table IV. Problem 4a performance comparisons.

\begin{tabular}{|c|c|c|c|c|c|}
\hline Case & Iterations & Total memory (GB) & Walltime (min) & Cores & $\begin{array}{c}\text { Time } \\
\text { (core-hr) }\end{array}$ \\
\hline Default & 12 & 46.85 & 54.23 & 29 & 26.21 \\
\hline ML Space & 12 & 46.75 & 42.28 & 29 & 20.44 \\
\hline ML Energy & 11 & 47.76 & 44.13 & 29 & 21.33 \\
\hline Subplane & 13 & 34.87 & 122.21 & 23 & 46.85 \\
\hline Subplane + ML Space & 17 & 34.70 & 105.15 & 23 & 40.31 \\
\hline Subplane + ML Energy & 12 & 35.73 & 76.29 & 23 & 29.24 \\
\hline
\end{tabular}

\section{CONCLUSIONS}

This paper presents an extension of the 2D/1D subplane method to encompass TH feedback based on the basis of subgrid solvers that were previously used for control rod and spacer grid axial heterogeneities. This approach reduces the computation burden of coupled MPACT/CTF calculations to make them more tractable for industry users while still providing a sufficient level of accuracy by making reasonable approximations. In general, the accuracy of the new approach is very good with roughly $0.6 \%$ maximum pin power error observed. In the single assembly case, negligible differences were observed in power and temperature distributions while still providing noteworthy speedup of $\sim 1.5-3 \times$. In the colorset case, accuracy was still good, but larger pin power errors were observed at a little over $0.5 \%$. This may be too high for a high-fidelity simulation capability, but future efforts can focus on minimizing this if possible through the use of the CPM-based subgrid solvers. Performance on the colorset, however, struggled with subplane, as the CMFD solve time increased. This area requires further work before the capability can be production-level, but the accuracy of the novel subgrid approach applied to temperature and density distributions is optimistic.

Future work will also focus on incorporating depletion into the capability. Initial efforts have already been performed, with room for improvement in terms of accuracy. However, this will be crucial for the next steps of working toward comparisons with operating reactors. 


\section{ACKNOWLEDGMENTS}

This research was supported by the Consortium for Advanced Simulation of Light Water Reactors (www.casl.gov), an Energy Innovation Hub (http://www.energy.gov/hubs) for Modeling and Simulation of Nuclear Reactors under US Department of Energy Contract No. DE-AC05-00OR22725.

This research made use of the resources of the Compute and Data Environment for Science (CADES) at Oak Ridge National Laboratory (ORNL), which is supported in part by the Office of Nuclear Energy of the US Department of Energy under Contract No. DE-AC05-00OR22725.

\section{REFERENCES}

1. B. Collins et al., "Stability and Accuracy of 3D Neutron Transport Simulations Using the 2D/1D Method in MPACT," Journal of Computational Physics, 326, 612 (2016).

2. J. Turner et al., "The Virtual Environment for Reactor Applications (VERA): Design and Architecture," Journal of Computational Physics, 326, 544 (2016)

3. S. Stimpson, A. Graham, and B. Collins. "Performance Improvements to the 2D/1D Subplane Method in MPACT," Proc. M\&C 2019, Portland, Oregon (August 25-29, 2019).

4. S. Stimpson, A. Graham, and B. Collins. "Subgrid Treatment of Spacer Grids in the 2D/1D Subplane Approach," Proc. M\&C 2019, Portland, Oregon (August 25-29, 2019).

5. M.N. Avramova, CTF: A Thermal Hydraulic Sub-Channel Code for LWR Transient Analyses, User's Manual. Technical Report, Pennsylvania State University, Department of Nuclear Engineering (2009).

6. A. Graham, "Subgrid Methods for Resolving Axial Heterogeneity in Planar Synthesis Solutions for the Boltzmann Transport Equation," PhD Dissertation, University of Michigan (2017).

7. A. Graham et al., "Subplane Collision Probabilities Method Applied to Control Rod Cusping in 2D/1D," Annals of Nuclear Energy, 118, pp. 1-14 (2018).

8. S. Stimpson, B. Collins, and B. Kochunas, "Improvement of Transport-Corrected Scattering Stability and Performance Using a Jacobi Inscatter Algorithm for 2D-MOC," Annals of Nuclear Energy, 105, pp. 1-10 (2017).

9. S. Stimpson, B. Collins, and T. Downar, "Axial Transport Solvers for the 2D/1D Scheme in MPACT," Proc. PHYSOR 2014, Kyoto, Japan (September 28-October 3, 2014).

10. J. Y. Cho et al., "Sub-plane scheme for a radial transport and axial diffusion code," Proceedings of the International Congress on Advances in Nuclear Power Plants (ICAPP) (2007).

11. A. Godfrey, "VERA Core Physics Benchmark Progression Problem Specifications," Revision 4, CASL-U-2012-0131-004, Revision 4, CASL, August 29, 2014. http://www.casl.gov/docs/CASL-U2012-0131-004.pdf.

12. L. Cornejo, B. Collins, S. Stimpson, "Multilevel CMFD Acceleration Methods for Whole Core Reactor Simulation in VERA," Proc. PHYSOR 2020, Cambridge, United Kingdom (March 29-April 2, 2020) 